\title{
Parallel-Connected Inverter Control Method in Material Micromachining
}

\author{
En-Chih Chang, Hongshu Huang, Wei Liu, and Rong-Ching Wu
}

\begin{abstract}
This paper proposes a parallel-connected inverter control method for material micromachining. The proposed control method is composed of a sigmoid sliding mode function (SSMF) and a firefly algorithm (FA). The SSMF is insensitive to system uncertainties, thus achieving high-quality AC output voltage. However, as the load of the micromachining (MM) is a step change situation, steady-state errors still exist in SSMF. The steady-state errors cause heat losses in transistors and high voltage harmonics in inverter output, and thus the stability and reliability of the MM are degraded. To attenuate steady-state errors, the control gains of the SSMF can be tuned via the FA. Using the proposed control method, the parallel-connected inverter yields a high-quality AC output voltage with low voltage harmonics and fast dynamic response. Experiments prove the applicability of the proposed control method in material MM. Since the proposed control method is easier to implement than previous control methods and allows high-precision tracking, this paper will be of interest to designers of correlative material micromachining.
\end{abstract}

Index Terms-Parallel-connected inverter, material micromachining, sigmoid sliding mode function (SSMF), firefly algorithm (FA), steady-state errors.

\section{INTRODUCTION}

Because the thermal management can be easily designed and the redundancy, modularity and maintainability are improved significantly, the parallel-connected inverters are popularly adopted in material MM [1]-[4]. The MM includes various roles such as drilling, engraving, and cutting that usually has the requirements of the precision and speed. The parallel-connected inverter of $\mathrm{MM}$ has to provide high-quality AC output voltage with low total harmonic distortion and fast dynamic response; these requirements can be obtained via closed-loop control methods. Some methodologies derived for inverter systems are developed, such as PID control, repetitive control, deadbeat control, and so on [5]-[10]. Though the PID control improves system performance under linear load situations, it can not yield good performance while the controlled plant is an uncertain

Manuscript received April 19, 2017; revised July 20, 2017. This work was supported by the Ministry of Science and Technology of Taiwan, R.O.C., under contract number MOST104-2221-E-214-011.

En-Chih Chang and Rong-Ching $\mathrm{Wu}$ are with the Department of Electrical Engineering, I-Shou University, No.1, Sec. 1, Syuecheng Rd., Dashu District, Kaohsiung City 84001, Taiwan, R.O.C. (e-mail: enchihchang@isu.edu.tw,rcwu@isu.edu.tw).

Hongshu Huang and Wei Liu are with the Electrical Engineering and Automation, Guangzhou University, Guangzhou 510006, P.R.C. (e-mail: hhs1550@163.com,13822109999@139.com). disturbance. Repetitive control and deadbeat control are capable of providing satisfactory system performance against severe nonlinear circumstances. However, the repetitive control has complicated control algorithms and makes digital realization difficult; deadbeat control is highly dependent on the precision of the parameters. Sliding mode control is developed in the 1950's that provides a robust methodology for governing nonlinear systems in the face of internal parameter variations and external disturbances [11]-[15]. The sliding mode control employs a switching control law to drive the controlled system to reach and sustain in the switching hyperplane. In order to design the sliding mode controller, there are two processes ad follows [16]-[20]:

(1) The trajectory of the state errors must be driven toward the sliding surface.

(2) As the state errors arrive at sliding surface, the trajectory of the state errors has to slide along the surface to the origin.

Recently, sigmoid sliding mode function (SSMF) has been proposed and is more insensitive to system uncertainties, and the SSMF controlled inverters can be found in the literature [21]-[25]. Using the SSMF to control parallel-connected inverter is thus a reasonable means. The SSMF has a voltage control loop, and such a loop is designed to obtain the output voltage with the desired amplitude and frequency among the parallel modules. However, the load of the MM is not fixed and may be a step change situation, and thus steady-state errors occur. The steady-state errors cause heat losses and high voltage harmonics, even incurs MM's instability and unreliability. Firefly algorithm (FA) is a meta-heuristic technology inspired by the sparkling behavior of fireflies, and thus has been used for resolving optimal control problems in various engineering fields [26]-[31]. To attenuate steady-state errors, the FA is well used to tune control gains of the SSMF. Therefore, by using the proposed control method, a closed-loop parallel-connected inverter yields good control performance in material MM even in face of step change load. Experiments are given to support the efficacy of the proposed control method. The paper is organized as follows: Section II describes the dynamic model of parallel-connected inverter, and the proposed control method is then derived. In Section III, the experimental results are performed in support of the proposed control method. Conclusions are given in Section IV.

\section{Proposed Control METHOD FOR} PARALLEL-CONNECTED INVERTER IN MATERIAL MM

Fig. 1(a) shows the parallel-connected inverter, which is composed of LC filter, switching component with MOSFET transistors and load. The equivalent circuit is displayed in Fig. 
1(b). From Fig. 1(b), the inverter dynamics can be written as

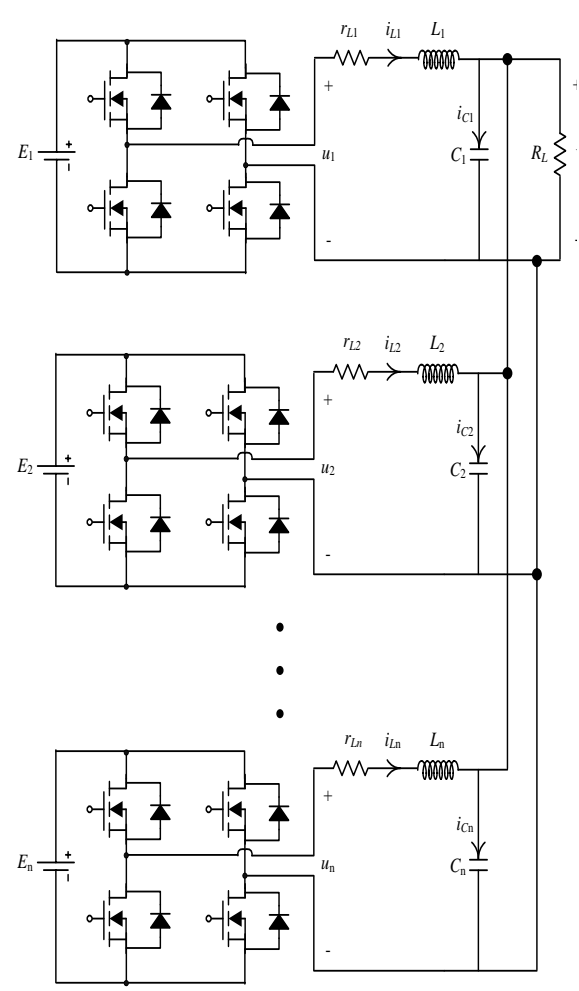

(a)

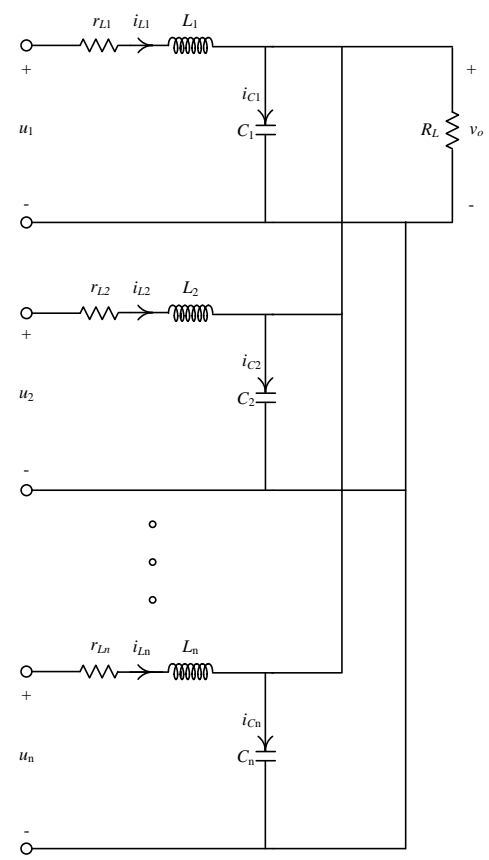

(b)

Fig. 1. Parallel-connected inverter.

$$
\left\{\begin{array}{c}
u_{1}=r_{L_{1}} i_{L_{1}}+L_{1} \frac{d i_{L_{1}}}{d t}+v_{O} \\
\cdot \\
\cdot \\
\cdot \\
u_{n}=r_{L_{n}} i_{L_{n}}+L_{n} \frac{d i_{L_{n}}}{d t}+v_{O} \\
i_{L_{1}}+i_{L_{2}}+\cdots+i_{L_{n}}=i_{C_{1}}+i_{O} \\
i_{C_{1}}=C_{t o t} \frac{d v_{O}}{d t}
\end{array}\right.
$$

where $C_{\text {tot }}=\sum_{i=1}^{n} C_{i}$.

Then, the state error $\tilde{x}$ between the output voltage and the reference voltage is expressed as

$$
\tilde{x}=v_{O}-v_{r}
$$

where $v_{O}$ is the output voltage, and $v_{r}=V_{m} \sin (\omega t)$ is the reference voltage.

Our objective is to design a control law well, thus providing output voltage regulation among the parallel modules. The control law, $u=u_{e}+u_{s}$ is derived as follows.

Suppose a sliding mode function be given by

$$
\sigma=c \tilde{X}
$$

where $c$ is constant.

The $u_{e}$ is only valid in the sliding surface as

$$
\left.\dot{\sigma}\right|_{u=u_{e}}=c \dot{\tilde{x}}=0
$$

By the use of the sliding mode reaching condition $\left.\sigma \dot{\sigma}\right|_{u=u_{e}+u_{s}}<0$, the existence of a sliding mode can be guaranteed, and thus $u_{S}$ can be obtained as

$$
u_{S}=-\operatorname{Ksgn}(\tilde{\sigma x})
$$

where $K$ is the control gain.

Notice that the (5) uses an sgn function and the chattering happens in practice. Therefore, the sgn function is replaced by the sigmoid sliding mode function as follows:

$$
f(x)=-\frac{2}{1+e^{-\gamma x}}+1
$$

where $\gamma$ stands for constant.

However, steady-state errors still exist in SSMF controlled system if a step change loading is applied. Thus, to attenuate steady-state errors, the FA is employed to tune the control gains of SSMF. Firstly, the sparkling properties of fireflies are idealized and three rules are assumed as follows:

(1) The whole fireflies are unisex and thus one firefly can be tempted at other fireflies, irrespective of sex.

(2) Attractiveness is in proportion to firefly sheen; less brilliant firefly moves towards more brilliant firefly. Once the distance increases among fireflies, the brilliance will decrease. The most brilliant firefly can randomly move because there are no insects to tempt it.

(3) The brilliance of the firefly relates to the analytic type of the cost function. For a maximization problem, the brilliance simplifies in proportional to the value of the cost function.

Secondly, when firefly $j$ is more attractive than firefly $i$ the movement is determined by the following equation:

$$
x_{i}=x_{i}+\alpha_{0} \cdot e^{-\beta r_{i j}^{2}} \cdot\left(x_{j}-x_{i}\right)+\delta \cdot(\text { rand }-0.5)
$$

where $\alpha_{0}$ is the firefly attractiveness value, $\beta$ denotes the media light absorption coefficient, $\delta \in[0,1]$, and rand represents random number between 0 and 1 . 
Finally in (7), the distance $r_{i j}$ between fireflies $i$ and $j$ can be obtained by

$$
r_{i j}=\sqrt{\sum_{k=1}^{D}\left(x_{i, k}-x_{j, k}\right)^{2}}
$$

Therefore, the FA compares the attractiveness of the firefly $i$ with the attractiveness of the firefly $j$. If the firefly $j$ is more attractive than firefly $i$, then the firefly $i$ is moved to the new position; otherwise the firefly $i$ will remain in the current position.

\section{EXPERIMENTAL RESULTS}

The system is tested for three inverter modules connected in parallel using the system parameters of the Table I.

\begin{tabular}{|c|r|}
\hline \multicolumn{2}{|c|}{ TABLE I: SYSTEM PARAMETERS } \\
\hline DC Voltage & $E_{1}=E_{2}=E_{3}=210 \mathrm{~V}$ \\
\hline Output Voltage & $110 \mathrm{~V}_{\text {rms }}$ \\
\hline Output Frequency & $60 \mathrm{~Hz}$ \\
\hline Filter Inductors & $L_{1} \cong L_{2} \cong L_{3} \cong 1.2 \mathrm{mH}$ \\
\hline Filter Capacitor & $C_{1}=15 \mu \mathrm{F}$ \\
\hline Inductor Resistances & $r_{L 1} \cong r_{L 2} \cong r_{L 3} \cong 0.1 \Omega$ \\
\hline Rated Load & \\
\hline
\end{tabular}

(a) Proposed control method (100V/div; 20A/div; 5ms/div).

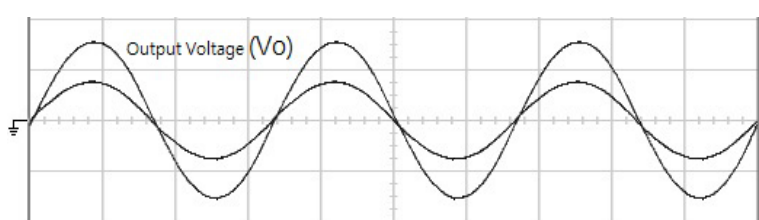

(b) Classic sliding mode function (100V/div; 20A/div; 5ms/div)

Fig. 2. Output waveshapes under resistive loading.

Fig. 2 (a) shows the output voltage and the load current for the proposed control method under full resistive load, and Fig. 2 (b) shows that for the classic sliding mode function under the same circumstance. Both output voltage are approximately sinusoidal waveshapes with ignorable distortion. Fig. 3 (a) and (b) show the output voltage and the load current obtained using proposed control method and classic sliding mode function under step load change from no load to full load, respectively. Investigation of the waveshapes reveals a smaller voltage sag in Fig. 3 (a), as compared with Fig. 3 (b). Fig. 4 plots the tracking errors under step load change. Clearly, the proposed control method reduces tracking errors, as compared to the classic sliding mode function. It is well proved that the proposed control method has better steady-state exactness, lower waveshape distortion and rapider convergence rate, as compared to the classic sliding mode function. The proposed control method is therefore more suitable for use in material MM. For further analysis of the Fig. 3 and Fig. 4, rapid steady-state recapture is seen and low output-voltage distortion shown in Fig. 3 (a) yields, as compared with Fig. 3 (b). This is because the proposed control method executes equal currents allotment among the inverter modules. In the Fig. 4, owing to the elimination of steady-state errors, the tracking errors obtained using proposed control method is quickly converged to zero but the classic sliding mode function controlled inverter exists in a large number of tracking errors. It is doubtless that the proposed control method is capable of offering wider requirements, such as machining of nanomaterials and nanocomposites, material measurement, cutting of composite materials, and thin film material manufacturing. Above-mentioned these will promote considerable future research.

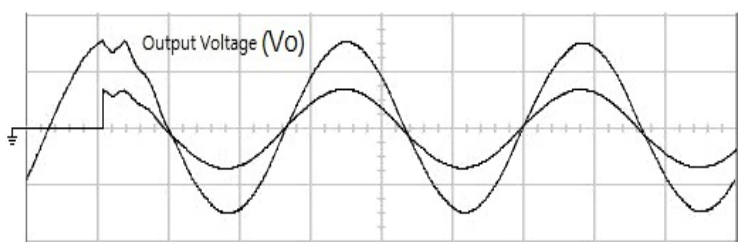

(a) Proposed control method (100V/div; 20A/div; 5ms/div).

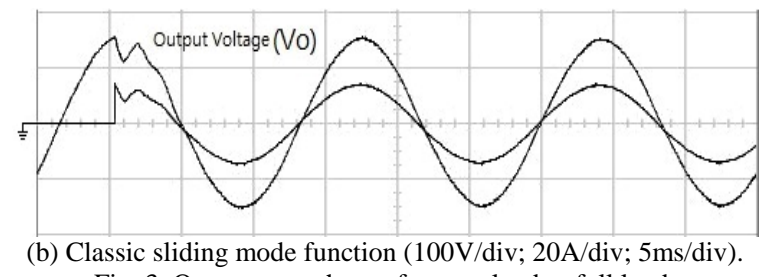
Fig. 3. Output waveshapes from no load to full load.

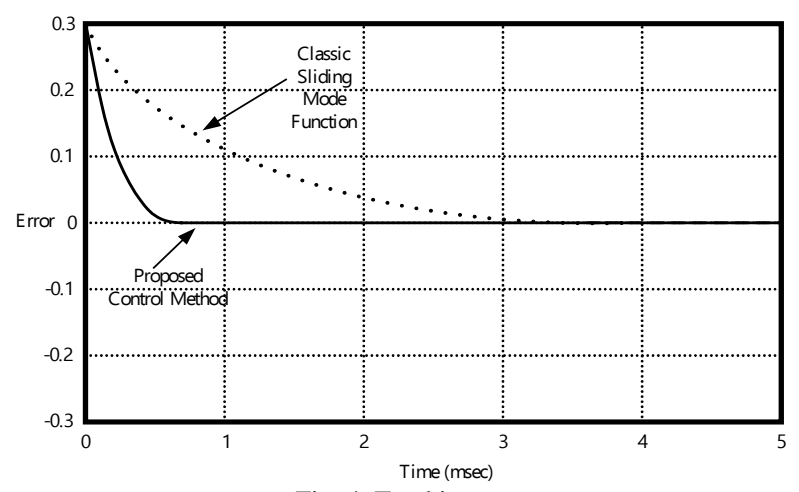

Fig. 4. Tracking errors.

\section{CONCLUSIONS}

This paper develops a high-performance parallel-connected inverter controlled material MM by associating SSMF with FA. The SSMF utilizes a voltage control loop to obtain AC output voltage regulation among the parallel modules. However, once the load of the MM is a highly nonlinear circumstance, steady-state errors around SSMF occurs. The FA is used to tune the control gains of the SSMF so that steady-state errors can be attenuated. 
Experimental results show the effectiveness and applicability of the proposed control method.

\section{REFERENCES}

[1] N. Mohan, T. M. Undeland, and W. P. Robbins, "Power electronics: converters," Applications, and Design, New York: Wiley, 2003.

[2] D. W. Hart, Power Electronics, New York: McGraw-Hill, 2010.

[3] Simon and O. Alejandro, Power-Switching Converters, FL: CRC Press, 2010.

[4] F. L. Luo and H. Ye, Power Electronics: Advanced Conversion Technologies, FL: CRC Press, 2010.

[5] J. Selvaraj and N.A. Rahim, "Multilevel inverter for grid-connected PV system employing digital PI controller," IEEE Trans. Ind. Electron., vol. 56, no. 1, pp. 149-158, 2009.

[6] R. Caceres, R. Rojas, and O. Camacho, "Robust PID control of a buck-boost UPS converter," in Proc. 2000 INTELEC, 2000, pp. 180-185.

[7] T. Hornik and Q. C. Zhong, "A current-control strategy for voltage-source inverters in microgrids based on $\mathrm{H} \infty$ and repetitive control," IEEE Trans. Power Electron., vol. 26, no. 3, pp. 943-952, 2011.

[8] K. Zhang, Y. Kang, J. Xiong, and J. Chen, "Direct repetitive control of SPWM inverter for UPS purpose,” IEEE Trans. Power Electron., vol. 18, no. 3, pp.271-280, 1995.

[9] X. G. Zhang, W. J. Zhang, J. M. Chen, and D. G. Xu, "Deadbeat control strategy of circulating currents in parallel connection system of three-phase PWM converter," IEEE Trans. Energy Conversion, vol. 29, no. 2, pp. 406-417, 2014

[10] T. Kawabata, T. Miyashita, and Y. Yamamoto, "Dead beat control of three-phase PWM inverter," IEEE Trans. Power Electron., vol. 5, no. 1 , pp. 21-28, 1990.

[11] U. Itkis, Control Systems of Variable Structure, New York: Wiley, 1976.

[12] V. I. Utkin, "Variable structure systems with sliding modes," IEEE Trans. Automatic Control, vol. AC-22, no. 2, pp. 212-222, 1977.

[13] H. Sira-Ramirez, "Sliding regimes in general non-linear systems: A relative degree approach,” Int. J. Contr., vol. 50, no. 4, pp. 1487-1506, 1989.

[14] Z. Doulgeri, "Sliding regime of a nonlinear robust controller for robot manipulators," IEEE Proceedings-Control Theory and Applications, vol. 146, no. 6, pp. 493-498, 1999.

[15] S. P. Stanchev, "A variant of an (combined) adaptive controller design introducing sliding regime in Lyapunov derivative,” in Proc. 2003 ACC Conf., 2003, pp. 909-914.

[16] L. Malesani, L. Rossetto, G. Spiazzi, and A. Zuccato, "An AC power supply with sliding-mode control,” in Proc. 1993 IAS Annual Meeting, 1993, pp. 623-629.

[17] C. Y. Chan, "Servo-system with discrete-variable structure control," Systems Control Letters, vol. 17, no. 4, pp. 321-325, 1991.

[18] S. L. Jung and Y. Y. Tzou, "Sliding mode control of a closed-loop regulated PWM inverter under large variations," in Proc. 1993 PESC, 1993, pp. 616-622.

[19] S. L. Jung and Y. Y. Tzou, "Discrete sliding-mode control of a PWM inverter for sinusoidal output waveform synthesis with optimal sliding curve," IEEE Trans. Power Electron., vol. 11, no. 4, pp. 567-577, 1996.

[20] T. L. Chern, J. Chang, C. H. Chen, and H. T. Su, "Microprocessor-based modified integral discrete variable-structure control for UPS," IEEE Trans. Power Electron., vol. 46, no. 2, pp. 340-348, 1999.

[21] L. G. Wu, P. Shi, and X. J. Su, Sliding Mode Control of Uncertain Parameter-Switching Hybrid Systems, New York: Wiley, 2014.

[22] A. T. Azar and Q. M. Zhu, Advances and Applications in Sliding Mode Control Systems, New York: Springer-Verlag, 2015.

[23] J. K. Liu and X. H. Wang, Advanced Sliding Mode Control for Mechanical Systems Design, Analysis and MATLAB Simulation, Berlin: Springer-Verlag, 2012.
[24] S. C. Tan, Y. M. Lai, and C. K. Tse, "Sliding mode control of switching power converters: techniques and implementation," FL: CRC Press, 2012.

[25] R. R. Ramos, B. Domingo, E. Fossas, and F. Guinjoan, "A fixed-frequency quasi-sliding control algorithm: application to power inverters design by means of FPGA implementation,” IEEE Trans. Power Electron., vol. 18, no. 1, pp. 344-355, 2003.

[26] J. Dreo, Metaheuristics for Hard Optimization, Berlin: Springer-Verlag, 2006.

[27] V. Pandian, Meta-Heuristics Optimization Algorithms in Engineering, Business, Economics, and Finance, Hershey: IGI Global, 2013.

[28] I. Rechenberg, Evolutionsstrategie, Stuttgart: Frommann-Holzboog, 1994.

[29] X. S. Yang, Nature-Inspired Metaheuristic Algorithms, Frome: Luniver Press, 2008.

[30] R. Tamilselvan and V. Padmathilagam, "Harmonic optimization in cascaded multi level inverter using firefly algorithm," Journal of Theoretical and Applied Information Technology, vol. 64, no. 3, pp. 773-780, 2014.

[31] B. Abderrahim, A. Mekki, and R. Imane, "Intelligent selection of machining parameters in multipass turnings using firefly algorithm," Modelling and Simulation in Engineering, vol. 2014, no. 8, pp. 1-6, 2014.

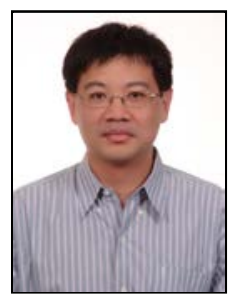

En-Chih Chang was born in Kaohsiung, Taiwan in 1975. He received his B.S. degree from Feng-Chia University, Taichung, Taiwan, R.O.C. in 1999, his M.S. degree from the National Taiwan Ocean University, Keelung, Taiwan, R.O.C. in 2001; and his $\mathrm{PhD}$ degree from the National Cheng Kung University, Tainan, Taiwan, R.O.C. in 2008, and all in electrical engineering. He is presently working as an associate professor in the Department of Electrical Engineering, I-Shou University, Kaohsiung, Taiwan, R.O.C.. His current research interests include sliding mode control, intelligent control, grey theory, and their applications in power electronics systems.

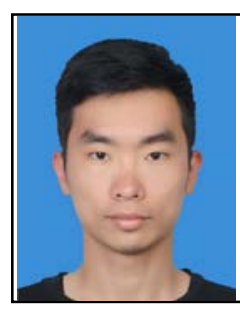

Hongshu Huang was born in Chaozhou, Guangdong, in 1995. He is working toward the B.S. degree in electrical engineering and automation from Guangzhou University. From September 7, 2016 to January 15, 2017, he is an exchange student in the Department of Electronic Engineering, I-Shou University, Kaohsiung City, Taiwan, R.O.C.. His further research interests include DC/AC inverter, power electronics, switching power supply, solar power supply, motor control and energy conservation.

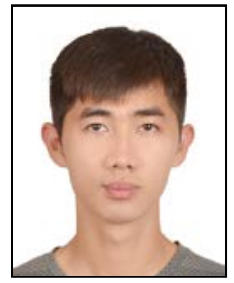

Wei Liu was born in Shaoguan, Guangdong in 1995. $\mathrm{He}$ is working toward the B.S. degree in electrical engineering and automation from Guangzhou University of China. From September 7, 2016 to January 15, 2017, he is an exchange student in Department of Electronic Engineering from I-Shou University, Kaohsiung City, Taiwan, R.O.C.. His further research interests include sliding mode control, industry electronic, new energy power generation.

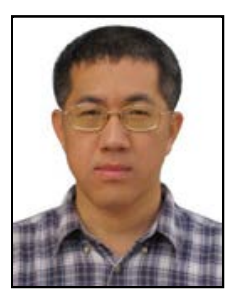

Rong-Ching Wu graduated from National Taiwan Institute of Technology, Taipei, Taiwan, in 1990. He received the M.Sc. degree in 1994 and received the $\mathrm{PhD}$ degree in 2001 from National Sun Yat-Sen University, Kaohsiung, Taiwan, R.O.C.. From 1991 to 2001, he was an electrical engineer in Taiwan Power Company, Kaohsiung, Taiwan, and was involved with design, construction, and operation of power systems. Currently, he is an associate professor at I-Shou University, Kaohsiung, Taiwan, R.O.C. 